\title{
Crystal structure of duck egg lysozyme isoform II (DEL-II)
}

\author{
David B. Langley ${ }^{1 *}$ (D) and Daniel Christ ${ }^{1,2}$
}

\begin{abstract}
Background: Lysozyme purified from duck eggs (DEL) has long been used as a model antigen as a counterpoint to the enzyme purified from hen eggs (HEL). However, unlike the single C-type variant found in hen eggs, duck eggs contain multiple isoforms: I, II and III. We recently reported the structures of isoforms I and III from Pekin duck (Anas platyrhynchos) and unequivocally determined the sequences of all three isoforms by mass spectrometry. Here we present the crystal structure of isoform II (DEL-II).

Results: Lysozyme isoform II was purified from isoforms I and III using ion-exchange and gel-filtration chromatography, then crystallized. X-ray diffraction data were collected to $1.15 \AA$ resolution and the structure of DEL-II was solved by molecular replacement using the structure of DEL-I as the search model. It contains two molecules in the crystallographic asymmetric unit: both molecules display a canonical C-type lysozyme fold and electron density consistent with the expected sequence. The most significant difference between the two molecules concerns different conformations of a surface loop containing one of the expected amino acid differences between the isoforms.

Conclusions: The structure of DEL-II supports the primary sequence as elucidated by a combination of amino acid sequencing, DNA sequencing and mass spectrometry, with strong electron density confirming it to be an S37G G71R variant of DEL I, and differing from hen egg lysozyme at a total of 21 amino acid positions.
\end{abstract}

Keywords: Duck egg lysozyme, Lysozyme, Structure, DEL-II

\section{Background}

While lysozymes purified from the eggs of chickens and ducks were both extensively studied throughout the 1960s, only the crystal structure of hen egg lysozyme (HEL) was reported [1]. This was no doubt aided by the fact that samples from duck eggs contained multiple isoforms (likely three), with amino acid analysis suggesting that isoforms were distinguished largely on the basis of containing different numbers of arginine residues [2, 3]. Although no structure of duck egg lysozyme (DEL) subsequently emerged, over the ensuing decades duck lysozyme was nevertheless intensely utilized in immunology. In particular, DEL was used as a model antigen juxtaposed against HEL, from which it differed at approximately 20 of the 129 amino acid positions ('approximately', as amino acid sequences derived via Edman

\footnotetext{
* Correspondence: d.langley@garvan.org.au

${ }^{1}$ Immunology Division, Garvan Institute of Medical Research, 384 Victoria

Road, Darlinghurst, Sydney, NSW 2010, Australia

Full list of author information is available at the end of the article
}

Degradation for different isoforms and from different strains by different groups disagreed at a handful of positions (see Langley et al., 2017 for a review)). Duck lysozyme, often strain and isoform unspecified, has been used to advance our understanding of many immunological phenomena including: antibody-antigen interactions [4, 5]; immune tolerance [6]; complement activation [7]; germinal center B-cell affinity maturation [8, 9]; T-follicular helper cell differentiation [10]; and, most recently, to dissect self/foreign discrimination during emergence from B-cell anergy [11].

The DNA sequence for one of the three Pekin duck isoforms (DEL-II) was finally published in 2013 [12], differing at two positions (both asparagine/aspartic acid discrepancies) with the sequence determined using Edman Degradation [13]. We recently used a combination of mass spectrometry and X-ray crystallography to unequivocally delineate sequences of all three Pekin DEL isoforms (DELs -I, -II and -III). However, we were only able to determine high-resolution X-ray structures of

(C) The Author(s). 2018 Open Access This article is distributed under the terms of the Creative Commons Attribution 4.0 International License (http://creativecommons.org/licenses/by/4.0/), which permits unrestricted use, distribution, and 
two isoforms (DEL-I and DEL-III) [14]. Here we report the high-resolution structure of the remaining isoform, DEL-II.

\section{Results}

The DEL-II structure comprises two C-type lysozyme molecules in the asymmetric unit (chains $-\mathrm{A}$ and $-\mathrm{B}$ ). The folds of the two molecules are highly similar to each other (root mean square deviation of $0.16 \AA$ over 99 CA positions) as well as to structures of DEL-I (PDB entry 5v8g) and DEL-III (PDB entrys 5v92 and 5v94) (see Fig. 1 for superpositions). The main difference between the folds of the chain-A and chain-B molecules are a surface loop (residues 67-73) which adopts a vastly different conformation within the chain- $B$ molecule, relative to the chain-A molecule and the folds of molecules within structures of DEL-I and DEL-III (Fig. 1). This alternate fold is reflected by substantial differences in backbone torsion angles for this region, as compared and highlighted in Additional file 1: Table S1.

The previously reported primary sequence of DEL-II, derived from amino acid and DNA sequencing as well as mass spectrometry data, predicted that it would be identical to that of DEL-I with the exception of two positions; S37G and G71R (Fig. 2, highlighted yellow) [14].

Negative peaks in $f_{\text {obs }}-f_{\text {calc }}$ difference maps covering the search model (DEL-I) clearly indicated the absence of a substantive side chain at position 37 (in both chains -A and -B), consistent with glycine at this position. Conversely, strong positive $f_{\text {obs }}-f_{\text {calc }}$ electron density clearly

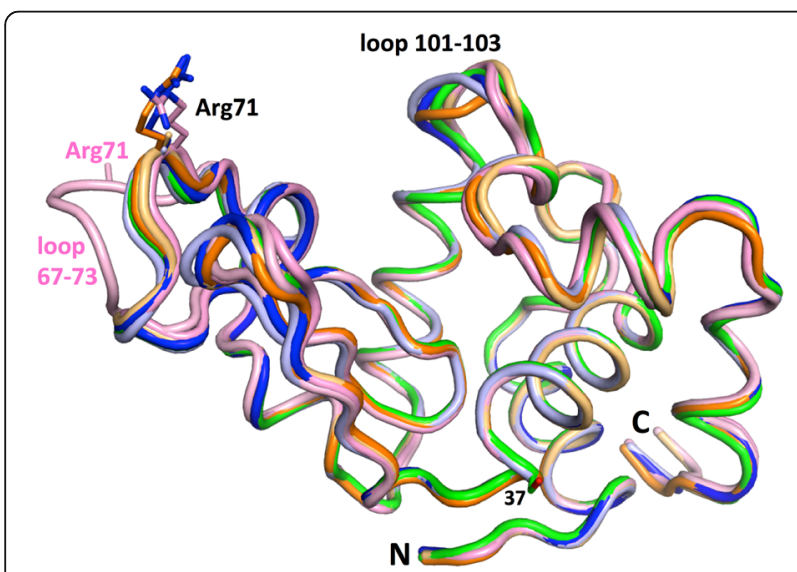

Fig. 1 Cartoon representation of DEL-II (chains -A and -B; pink and light pink) as viewed down the catalytic cleft and superposed with DEL-I (PDB entry 5v8g, green) and DEL-III (two crystal forms: PDB entry $5 \mathrm{~V} 92$ (orthorhombic form) coloured orange and light orange (chains -A and -B); and PDB entry 5 V94 (cubic form) coloured blue and light blue (chains -A and -B)). Positions 37 and 71 are shown as sticks. The structures differ most in loop regions; loop101-103, and loop 67-73. DEL-II chain B differs most starkly from the ensemble at loop 67-73 (light pink loop and text), including Arg71, the side chain of which is disordered indicated a well-ordered arginine side chain at position 71 of the A-chain (this side chain was disordered within the B-chain molecule), confirming that DEL-II is indeed an S37G G71R double mutant of DEL-I (composite omit maps shown in Fig. 3). As for DEL-I, DEL-II lacked the additional two arginine residues located at positions 79 and 100, which are observed in DEL-III (Fig. 3) [14].

Although the quality of the electron density maps was generally extremely high (as expected at $1.15 \AA$ resolution), there were a handful of side-chains that could not be resolved which have subsequently not been modeled (chain-A residues 47, 125 and 128; chain-B residues $45,47,68,71,122)$. Additionally, the electron density maps included several 'blob' features that we have not attempted to model (near residues Arg85/Asp87 of the A-chain, and Asn19 of the B-chain) adjacent residues clearly modeled in alternate conformations. $\mathrm{A} \mathrm{Mg}^{2+}$ ion and surrounding coordinated water ligands (octahedral geometry) has been modeled adjacent residue Glu35 in both A- and B-chains. A handful of $\mathrm{Cl}^{-}$ions have also been modeled, their identity confirmed by strong residual $f_{\text {obs }}-f_{\text {calc }}$ peaks on top of modeled water, generally large distances to adjacent ligands $(\sim 3.0-3.2 \AA)$ and weak but discernable peaks above noise in anomalous difference maps, of magnitude similar to peaks coincident with sulfur atoms present in cysteine and methionine residues (see pink mesh in Fig. 4). Both $\mathrm{Mg}^{2+}$ and $\mathrm{Cl}^{-}$ions were present in the crystallization conditions ( $100 \mathrm{mM} \mathrm{MgCl}_{2}$ after dilution).

\section{Discussion}

The structure of Pekin duck egg lysozyme isoform II (DEL-II) outlined here confirms prior sequencing and mass spectrometry data, and completes our previous structural studies of isoforms I and III [14]. Although the overall fold of DEL-II corresponds to a classical C-type lysozyme, as expected, in one of the two molecules of the asymmetric unit a surface loop of the B-chain molecule (residues 67-73) differs significantly from the A-chain molecule and from DEL-I and DEL-III, which are typical of the classic HEL fold. Centrally located within this loop is one of the four residues that together distinguish DELs -I, -II and -III, position 71. Arginine 71 in DEL-II (glycine in DEL-I) mediates interactions between the chain-A and $-B$ molecules: the guanidinium side group of the chain-A molecule stacks against the guanidinium side chain of Arg97 of the chain-B molecule, hydrogen-bonds the side chain of Asp101 of chain-B, and it's $\mathrm{N} \varepsilon$ atom coordinates an adjacent $\mathrm{Cl}^{-}$ion (distance $~ 3.17 \AA$ ) held at an intermolecular interface (Fig. 4). In contrast, the side chain of Arg71 in the B-chain molecule is not observed at all in the electron density, consistent with this residue being part of a loop that packs differently within the crystal lattice 


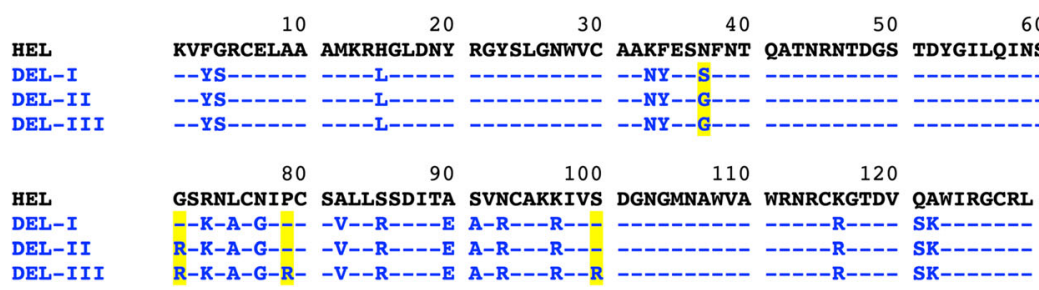

Fig. 2 Alignment of predicted sequences of DELS -I, -II and -III (blue text) in comparison with HEL (black text), as determined by amino acid sequencing [13], DNA sequencing [12] and mass spectrometry [14]. Sequence positions identical to HEL are indicated by a dash. DEL-II is an S37G G71R double mutant of DEL-I and a R79P R100S double mutant of DEL-III (positions highlighted yellow)

compared with the A-chain molecule and with other DEL structures (Fig. 1, loop 67-73). The electron density for this B-chain loop region is weak compared to the A-chain molecule (average CA atomic B-factors of 22.7 and $12.0 \AA^{2}$, respectively, compared with 12.8 and

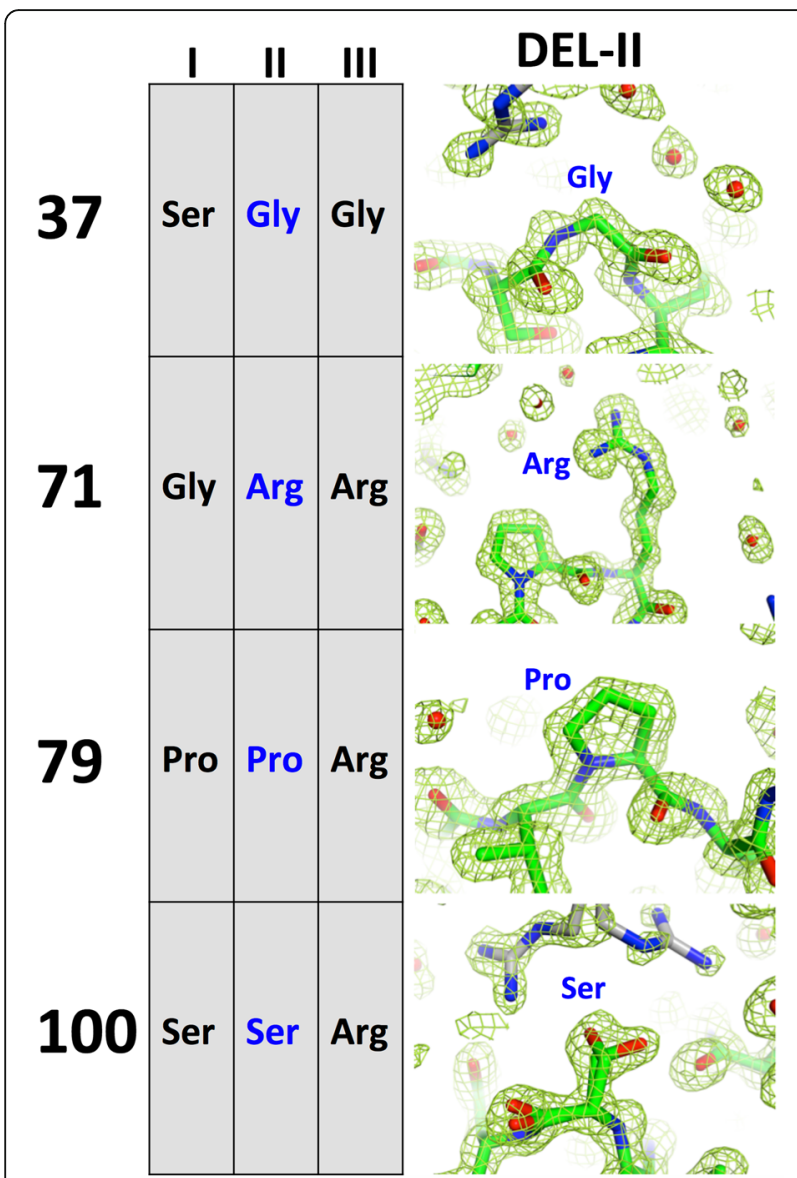

Fig. 3 Crystallographic confirmation of DEL-II sequence. The four amino acid positions at which Pekin DEL isoforms differ (columns on the left) are compared with composite omit map electron density for the DEL-II structure (RHS, contoured at 1 standard deviation above the mean). Sticks coloured grey belong to symmetry related molecules within the crystal lattice. Sequences corresponding to the DEL-II isoform are coloured blue. At position 100, multiple conformers of Ser100 were refined (adjacent multiple conformers of Arg114 within a neighboring molecule)
$11.4 \AA^{2}$ for the full-length $\mathrm{B}$ - and A-chain molecules (Table 2)), with the residue at the apex of the loop (Pro70) slotting between the side chain of Asn103 of the A-chain molecule and the side chains of Asp87 and Thr89 of a symmetry-related A-chain molecule within the crystal lattice. The neighboring residue, Thr69, contributes the only direct hydrogen bonds from this loop (B-chain Thr69 $\mathrm{O}$ atom) to symmetry-related A-chain Arg14 atoms $\mathrm{N} \varepsilon$ and NH2. These DEL-II B-chain molecular contacts and mode of crystal packing are not employed in the DEL-I crystal where position 71 is replaced by a glycine residue, which, in the context of the DEL-I crystal, contributes no main-chain hydrogen bonds to crystal packing. Although the conformation of this loop is similar in DEL-II chain-A to molecules within the two crystal forms of DEL-III, the crystal contacts are, again, distinct. In both DEL-III crystal forms

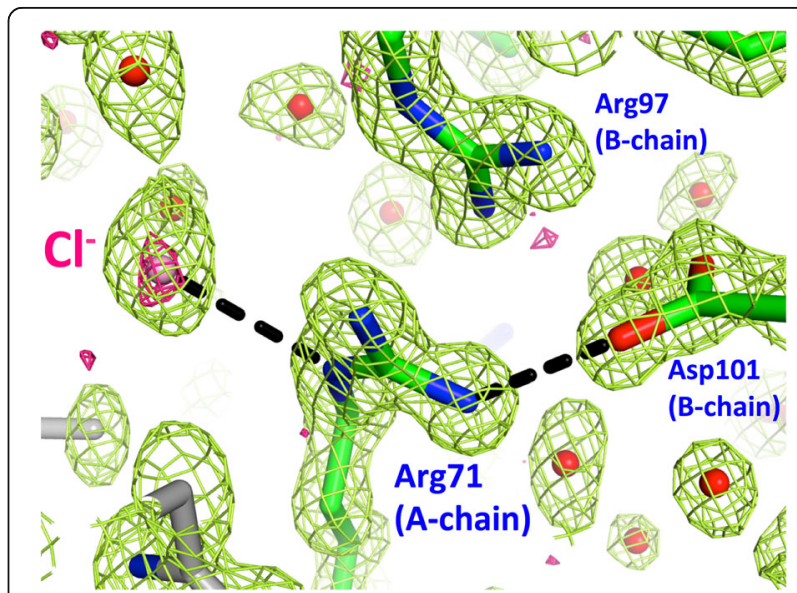

Fig. 4 Arg71 supports different crystal packing in DEL-II relative to DEL-I. Arg71 of DEL-II projects from the A-chain molecule and interacts with the B-chain molecule by packing against Arg97 and hydrogen-bonding Asp101. It also coordinates a chloride ion (pink ball and mesh), which sits at the interface between neighboring molecules. The green mesh represents composite omit map electron density (contoured at 1 standard deviation above the mean), whilst pink mesh represents an anomalous difference map (contoured at 3 standard deviations above the mean). Sticks coloured grey belong to a symmetry related molecule within the crystal lattice 
the side chain of Arg71 is well resolved in the A-chain molecules (whilst disordered in the B-chain molecules, which otherwise maintain the same fold), and in neither cases (for A- or B- chains) does the residue make any direct hydrogen bonds to neighboring molecules within the crystals. The diversity of fold noted for loop 67-73 (and for loop 101-103, and the C-terminus (Fig. 1)), is not uncommon within crystal structures on the surfaces of proteins, within loops connecting helices or sheets, or at polypeptide termini.

The unit cells of crystal forms of DELs -I and -II are somewhat related in that one of the unit cell dimensions is roughly equivalent ( $\mathrm{a} \sim 27 \AA$, both space groups are $P 2_{1}$ ). We suspect that earlier attempts to obtain a DEL-II structure (scrappy interleaved crystals, poorly defined cell dimensions, multiple weak molecular replacement solutions, unconvincing maps and stalled refinement) were symptomatic of significant amounts of DEL-I contaminating the DEL-II sample and distorting lattice growth. During purification, salt-gradient mediated elution of DELs off CM resin results in DELs -I and -II partially overlapping. In terms of charge, DEL-I and DEL-II differ by just a single positive charge in the form of Arg71. Discarding DEL-II-containing fractions adjacent to those of the DEL-I peak (to three quarters peak height) presumably sufficiently removed contamination with DEL-I, allowing high-quality DEL-II crystals to be grown.

The structure of DEL-II presented is also consistent with our previous analysis of interactions between the Pekin DEL isoforms and the landmark anti-lysozyme antibodies HyHEL5 [4] and HyHEL10 [15] to which, as with DEL-I (but not DEL-III), DEL-II binds with high $\left(\mathrm{K}_{\mathrm{D}} \sim 40 \mathrm{nM}\right)$ affinity [14].

\section{Conclusions}

Duck Egg Lysozyme (DEL) has been used as an important immunological model antigen since the 1960s. However, the exact sequences of the multiple DEL isoforms had remained ambiguous. We have recently confirmed the primary sequence of all three Pekin DEL isoforms and have reported high-resolution structures of DEL-I and -III [14]. Here we present the high-resolution structure of the remaining DEL-II isoform. Our study confirms that DEL-II corresponds to an S37G G71R variant of DEL-I, thereby completing the structural characterization of all three DEL isoforms.

\section{Methods}

\section{Purification and crystallization}

DEL-II was purified from DELs -I and -III, essentially as previously described [14]. Specifically, the protein was eluted from carboxy-methyl (CM) ion-exchange resin using a salt gradient $(50-450 \mathrm{mM} \mathrm{NaCl})$ in $50 \mathrm{mM}$ Tris (pH 8.8) buffer. As elution peaks corresponding to DELs
-I and -II (that elute sequentially) partially overlap, early fractions of the DEL-II peak (to about three quarters peak height) were discarded. Pooled fractions containing the remainder of the DEL-II peak were further chromatographed on a S200 26/60 gel-filtration column using a running buffer comprising $25 \mathrm{mM}$ Tris ( $\mathrm{pH}$ 8.0), $150 \mathrm{mM} \mathrm{NaCl}$. Fractions containing DEL-II were pooled and concentrated to $\sim 6 \mathrm{mg} / \mathrm{mL}$. Crystallization conditions were established using a Mosquito liquid handling robot which combined $400 \mathrm{~nL}$ well solution with $400 \mathrm{~nL}$ protein solution using the commercial PACT premier sparse matrix screen (Molecular Dimensions). After incubation at room temperature for 1 week, plate-like crystals appeared in condition D10 (well solution comprising $200 \mathrm{mM} \mathrm{MgCl} 2,100 \mathrm{mM}$ Tris (pH 8.0) and 20\% (v/v) PEG6000. Crystals were removed with a nylon loop and flash frozen by plunging the loop into liquid nitrogen.

\section{Structure solution and refinement}

X-ray diffraction data (Table 1) were recorded at the Australian Synchrotron on beamline MX2 using an Eiger $\times 16 \mathrm{M}$ detector (Dectris). A 360 degree sweep of data was deconvoluted into 3600 images (0.1 degree each), from which reflections were indexed and integrated using iMOSFLM [16]. The space group was determined with POINTLESS [17], and scaling was performed with AIMLESS [18], both part of the CCP4 suite of software. The structure was solved by molecular replacement using PHASER [19], where 2 molecules of DEL-I (PDB entry $5 \mathrm{v} 8 \mathrm{~g}$ ) were placed in the asymmetric unit (only $32 \%$ solvent). Rigid body and rounds of restrained B-factor refinement were performed with REFMAC5

Table 1 Data collection and processing

\begin{tabular}{|c|c|}
\hline Crystal & DEL-II \\
\hline Diffraction source & MX2, Australian Synchrotron \\
\hline Wavelength $(\AA)$ & 0.9537 \\
\hline Spacegroup & $P 2_{1}$ \\
\hline $\begin{array}{l}\text { Unit cell dimensions: } a, b, c \\
(\AA) ; a, \beta, \gamma_{1}\left(^{\circ}\right)\end{array}$ & $27.34,55.13,69.03 ; 90.00,95.95,90.00$ \\
\hline Resolution range & $42.99-1.15(1.17-1.15)$ \\
\hline Total reflections & $464,884(21,959)$ \\
\hline Unique reflections & 72,395 (3563) \\
\hline Completeness (\%) & $100(100)$ \\
\hline Multiplicity & $6.4(6.2)$ \\
\hline Average $(I / \sigma(I))$ & $9.0(2.9)$ \\
\hline Mean half set correlation, $\mathrm{CC}_{(1 / 2)}$ & $0.998(0.624)$ \\
\hline Rmeas (all I+ and I-) & $0.102(0.739)$ \\
\hline Rpim (all I+ and I-) & $0.039(0.291)$ \\
\hline Wilson $B\left(\AA^{2}\right)$ & 7.0 \\
\hline
\end{tabular}

Data within parentheses are for the highest resolution shell 
Table 2 Structure solution and refinement statistics

\begin{tabular}{ll}
\hline Crystal & DEL-II \\
\hline$R_{\text {work }} / R_{\text {free }}$ & $0.155 / 0.186$ \\
Molecules/asu & 2 \\
Atoms protein & 2074 \\
B average protein $\left(\AA^{2}\right)$ & A chain, 11.4; B chain, 12.8 \\
Atoms water & 280 \\
B average water $\left(\AA^{2}\right)$ & 22.2 \\
Atoms ions & $2 \times \mathrm{Mg}^{2+}, 5 \times \mathrm{Cl}^{-}$ \\
B average ions $\left(\AA^{2}\right)$ & $\mathrm{Mg}^{2+}, 17.6 ; \mathrm{Cl}^{-}, 16.0$ \\
RMSD bond lengths $(\AA)$ & 0.011 \\
RMSD bond angles ( $\left.{ }^{2}\right)$ & 1.6 \\
Ramachandran Outliers (\%) & 0 \\
Ramachandran Favored (\%) & 99.2 \\
PDB entry & $6 \mathrm{~d} 9 \mathrm{i}$ \\
\hline
\end{tabular}

[20], interspersed with inspection of models and maps enabling the correction of sequence and addition of solvent components using COOT [21]. Model refinement statistics are shown in Table 2. The high resolution of the data permitted use of anisotropic B-factors. Model validation was performed using the MOLPROBITY web server [22].

\section{Additional file}

Additional file 1: Table S1. Backbone torsion angles for DEL-II molecules chain- $A$ and-B, residues $60-80$. Angles which differ by greater than 20 degrees between chains-A and-B are shaded yellow. (DOCX $146 \mathrm{~kb}$ )

\section{Abbreviations}

DEL: Duck egg lysozyme; DEL-I: Duck egg lysozyme isoform I; DEL-II: Duck egg lysozyme isoform II; DEL-III: Duck egg lysozyme isoform III; HEL: Hen egg lysozyme; $K_{D}$ : Equilibrium binding constant; PEG: Polyethylene glycol

\section{Acknowledgements}

We thank Mary Christie for collecting the dataset. We thank the staff of the Australian Synchrotron, Beamline MX2.

\section{Funding}

This work was supported by National Health and Medical Research Council (NHMRC) Program Grant 1113904 and Australian Research Council (ARC) Discovery Grant 160104915.

\section{Availability of data and materials}

The atomic coordinates and structure factors for the structure have been deposited in the Protein Data Bank with accession code $6 \mathrm{~d} 9 \mathrm{i}$

\section{Authors' contributions}

DBL performed protein purification, crystallography and wrote the manuscript. DC directed research and wrote the manuscript. Both authors read and approved the final manuscript.

\section{Ethics approval and consent to participate}

Ethics approval was not required as only duck eggs were used as the source of protein

\section{Consent for publication}

Not applicable.

\section{Competing interests}

The authors declare that they have no competing interests.

\section{Publisher's Note}

Springer Nature remains neutral with regard to jurisdictional claims in published maps and institutional affiliations.

\section{Author details}

${ }^{1}$ Immunology Division, Garvan Institute of Medical Research, 384 Victoria Road, Darlinghurst, Sydney, NSW 2010, Australia. ${ }^{2}$ The University of New South Wales, Faculty of Medicine, St Vincent's Clinical School, Darlinghurst, Sydney, NSW 2010, Australia.

Received: 22 May 2018 Accepted: 7 August 2018

Published online: 22 August 2018

\section{References}

1. Blake CC, Koenig DF, Mair GA, North AC, Phillips DC, Sarma VR. Structure of hen egg-white lysozyme. A three-dimensional Fourier synthesis at 2 angstrom resolution. Nature. 1965;206(4986):757-61.

2. Jolles J, Spotorno G, Jolles P. Lysozymes characterized in duck egg-white: isolation of a histidine-less lysozyme. Nature. 1965;208(5016):1204-5.

3. Jolles J, Hermann J, Niemann B, Jolles P. Differences between the chemical structures of duck and hen egg-white lysozymes. Eur J Biochem. 1967;1(3): 344-6.

4. Smith-Gill SJ, Wilson AC, Potter M, Prager EM, Feldmann RJ, Mainhart CR. Mapping the antigenic epitope for a monoclonal-antibody against lysozyme. J Immunol. 1982;128(1):314-22.

5. Lavoie TB, Drohan WN, Smith-Gill SJ. Experimental-analysis by site-directed mutagenesis of somatic mutation effects on affinity and fine specificity in antibodies specific for lysozyme. J Immunol. 1992;148(2):503-13.

6. Shokat KM, Goodnow CC. Antigen-induced B-cell death and elimination during germinal-center immune-responses. Nature. 1995;375(6529):334-8.

7. Manderson AP, Quah B, Botto M, Goodnow CC, Walport MJ, Parish CR. A novel mechanism for complement activation at the surface of B cells following antigen binding. J Immunol. 2006;177(8):5155-62.

8. Allen CDC, Okada T, Tang HL, Cyster JG. Imaging of germinal center selection events during affinity maturation. Science. 2007;315(5811):528-31.

9. Phan TG, Green JA, Gray EE, Xu Y, Cyster JG. Immune complex relay by subcapsular sinus macrophages and noncognate B cells drives antibody affinity maturation. Nat Immunol. 2009:10(7):786-U153.

10. Lee JY, Skon CN, Lee YJ, Oh S, Taylor JJ, Malhotra D, Jenkins MK, Rosenfeld MG, Hogquist KA, Jameson SC. The transcription factor KLF2 restrains CD4 (+) T follicular helper cell differentiation. Immunity. 2015;42(2):252-64.

11. Burnett DL, Langley DB, Schofield P, Hermes JR, Chan TD, Jackson J, Bourne K, Reed JH, Patterson K, Porebski BT, et al. Germinal center antibody mutation trajectories are determined by rapid self/foreign discrimination. Science. 2018;360(6385):223-6.

12. Huang YH, Li YR, Burt DW, Chen HL, Zhang Y, Qian WB, Kim H, Gan SQ, Zhao YQ, Li JW, et al. The duck genome and transcriptome provide insight into an avian influenza virus reservoir species. Nat Genet. 2013;45(7):776.

13. Kondo K, Fujio H, Amano T. Chemical and immunological properties and amino-acid-sequences of 3 lysozymes from Peking-duck egg-white. J Biochem Tokyo. 1982;91(2):571-87.

14. Langley DB, Crossett B, Schofield P, Jackson J, Zeraati M, Maltby D, Christie M, Burnett D, Brink R, Goodnow C, et al. Structural basis of antigen recognition: crystal structure of duck egg lysozyme. Acta Crystallogr Sec D. 2017;73:910-20.

15. Silverton EW, Padlan EA, Davies DR, Smithgill S, Potter M. Crystalline monoclonal-antibody Fabs complexed to hen egg-white lysozyme. J Mol Biol. 1984;180(3):761-5.

16. Battye TGG, Kontogiannis L, Johnson O, Powell HR, Leslie AGW. iMOSFLM: a new graphical interface for diffraction-image processing with MOSFLM. Acta Crystallogr D. 2011;67:271-81.

17. Evans PR. An introduction to data reduction: space-group determination, scaling and intensity statistics. Acta Crystallogr D. 2011;67:282-92.

18. Evans PR, Murshudov GN. How good are my data and what is the resolution? Acta Crystallogr D. 2013;69:1204-14. 
19. McCoy AJ, Grosse-Kunstleve RW, Adams PD, Winn MD, Storoni LC, Read RJ. Phaser crystallographic software. J Appl Crystallogr. 2007;40(Pt 4):658-74

20. Murshudov GN, Skubak P, Lebedev AA, Pannu NS, Steiner RA, Nicholls RA, Winn MD, Long F, Vagin AA. REFMAC5 for the refinement of

macromolecular crystal structures. Acta Crystallogr D. 2011;67:355-67.

21. Emsley P, Lohkamp B, Scott WG, Cowtan K. Features and development of Coot. Acta Crystallogr D. 2010;66:486-501.

22. Chen VB, Arendall WB 3rd, Headd JJ, Keedy DA, Immormino RM, Kapral GJ, Murray LW, Richardson JS, Richardson DC. MolProbity: all-atom structure validation for macromolecular crystallography. Acta Crystallogr D Biol Crystallogr. 2010;66(Pt 1):12-21.

Ready to submit your research? Choose BMC and benefit from:

- fast, convenient online submission

- thorough peer review by experienced researchers in your field

- rapid publication on acceptance

- support for research data, including large and complex data types

- gold Open Access which fosters wider collaboration and increased citations

- maximum visibility for your research: over $100 \mathrm{M}$ website views per year

At BMC, research is always in progress.

Learn more biomedcentral.com/submissions 